\title{
FRONTERAS INTERNACIONALES, RECURSOS NATURALES E INTEGRACIÓN REGIONAL EN EL CONO SUR DE AMÉRICA DEL SUR
}

\begin{abstract}
Alejandro Schweitzer ${ }^{1}$
\section{Resumo}

$\mathrm{O}$ artigo trata de tendências de transformações recentes em espaços fronteiriços principalmente na Bacia do Prata, entre Brasil, Argentina, Paraguai e Uruguai, e na região transfronteiriça da Patagônia Austral, entre Argentina e Chile analisando as fronteiras e expansões geográficas; o lugar dos recursos naturais como fatores estratégicos no contexto da crise no século 21; a disputa por ouro, prata, petróleo e água e as repercussões dos processos de acumulação, despossessão e expropriação dos recursos naturais nas fronteiras como espaços de acumulação e resistência. As fronteiras a que se refere o artigo são, em quase todos os casos, espaços periféricos em relação aos seus respectivos centros nacionais, onde seguem sendo predominantes o modelo primário-exportador e extrativista. Os recursos naturais em disputa nas grandes regiões analisadas são o ouro, a prata, o petróleo e a água. Por fim, o artigo ressalta a inexistência de resistência em escala macrorregional transfronteiriça para fazer frente aos efeitos negativos da exploração de recursos naturais.
\end{abstract}

Palavras-chave: Espaço Fronteiriço. Bacia do Prata. Patagônia Austral. Expansão Geográfica. Exploração de Recursos Naturais.

\begin{abstract}
The article deals with trends of recent changes in border areas especially in the La Plata Basin, between Brazil, Argentina, Paraguay and Uruguay, and in the border region of southern Patagonia between Argentina- Chile and analyzing borders and geographic expansions; the place of natural resources as strategic factors in the context of crisis in the 21 st century; the dispute for gold, silver, oil and water and the impacts of the processes of accumulation, dispossession and expropriation of natural resources across borders as spaces of resistance and accumulation. The boundaries referred to in the article are, in almost all cases, peripheral areas in relation to their respective national centers, where continues to be predominant the export model of natural goods and extraction. Natural resources in dispute in the major regions analyzed are gold, silver, oil and water. Finally, the article highlights the lack of cross resistance in macro-regional level to tackle the negative effects of exploitation of natural resources.
\end{abstract}

Keywords: Border Area. The La Plata Basin. Southern Patagonia. Geographic Expansion. Exploitation of Natural Resources.

\section{INTRODUCTION}

El presente trabajo se inscribe en el marco de una línea de investigación colectiva, con sede en el Centro de Estudios Urbanos y Regionales, desde mediados de la década del 80 sobre las fronteras internas del MERCOSUR A4 (Argentina, Brasil, Paraguay y Uruguay), con mayor énfasis en lo personal desde mediados de la década siguiente sobre espacios de la Triple Frontera del Iguazú y Posadas/Encarnación y desde hace ocho años, ya coordinando un equipo de investigación de diferentes disciplinas, sobre las fronteras patagónicas australes entre Argentina y Chile.

\footnotetext{
${ }^{1}$ Arquiteto. Doutor em Geografia pela Universidade Paris III. Universidade Nacional Patagônia Austral, Rio Gallegos, Argentina. http://www.unpa.edu.ar/node/9 Email: alejandro.schweitzer@gmail.com
} 
En todos estos espacios salvo escasas excepciones, las actuales fronteras internacionales son heredadas del periodo colonial y asentadas físicamente sobre ríos y cadenas montañosas ${ }^{2}$. Ya desde mediados del Siglo XIX en el espacio misionero y desde fines de ese mismo siglo en el patagónico austral se hizo evidente que esos soportes de frontera lo eran a su vez de actividades productivas, extractivas unas, ganaderas o agrícolas otras y, en el caso de la Cuenca del Plata, a su vez, al menos hasta la extensión de las redes ferroviarias en el siglo siguiente, principales ejes de transporte.

Actualmente en estos espacios fronterizos, que en casi todos los casos son periféricos respecto a los respectivos centros nacionales, sigue siendo predominante el modelo primarioexportador y extractivo y, justamente por sus características periféricas, en general de menor atención por parte de estados nacionales, con modalidades muchas veces de aprovechamiento no sustentable, de depredación y explotación irracional de recursos inicialmente sustentables.

En todos los casos e independientemente de sus situaciones fronterizas, donde asumen características particulares, la realización de las actividades productivas depende de las condiciones de producción del espacio geográfico, garantizadas en el marco de procesos de expansión de capitales insertos en dinámicas mundiales afectadas desde 2008 por la crisis financiera global. Esta crisis coincide temporalmente y se asocia con la crisis ecológica, producto del cambio climático originado en el consumo excesivo de recursos naturales, se trata de hecho de una sola crisis sistémica.

Es por eso que en el análisis de los procesos productivos en espacios transfronterizos cobra particular interés el tratamiento de las modalidades de explotación de los recursos naturales, en particular minerales metalíferos, petróleo y agua.

El presente trabajo se estructura en tres partes. En primer lugar se exponen algunas consideraciones generales sobre el concepto de espacio geográfico, poniéndolas enseguida en relación con las interacciones en espacios fronterizos y los procesos de expansión geográfica, a fin de sintetizar a manera de hipótesis un posible perfil metodológico para el estudio de fronteras.

En segundo lugar estos mismos elementos (fronteras y recursos naturales) son enmarcados en el escenario actual de la crisis, presentando particularmente el papel estratégico de los recursos naturales, los procesos de acumulación por desposesión que permitieron en algunos casos la transferencia de recursos y valores de uso colectivo del sector público al privado y las transferencias de excedentes entre sociedades regionales, empresas y estados y exponiendo los casos concretos de los recursos oro, petróleo y agua.

En tercer lugar, a manera de conclusión, se presentan algunas consideraciones en torno a las fronteras como espacios de acumulación por parte de las empresas dominantes en los

\footnotetext{
${ }^{2}$ La excepción más significativa es la de la experiencia jesuítica que desde 1609 hasta 1767 ocupó el actual espacio fronterizo de Misiones tomando como frontera espacios "vacíos", de difícil acceso y tránsito, como son hasta la actualidad los esteros de Neembucú y del Iberá en Paraguay y Argentina respectivamente y con límites difusos en la Selva paranaense hacia el Brasil, mientras que los ejes principales de vinculación eran los actuales ríos Paraguay, Paraná y Uruguay, que luego marcaron las fronteras (Schweitzer, A. 2000).
} 
procesos de explotación de recursos naturales y espacios de resistencia de los movimientos sociales, así como las desigualdades en poder y organización entre las primeras y los segundos, mostrando la necesidad de aportar hacia una definitiva integración de los pueblos, como única alternativa para contrarrestar efectos negativos de la explotación de recursos naturales y potenciar los que sean positivos.

Finalmente, cabe aclarar que las consideraciones que se vierten en este documento pretenden ser apenas hipótesis de interpretación. Por un lado, debido a la novedad de algunos procesos y las características recientes, todavía no consolidadas, de algunas iniciativas que se presentan. Por otro lado, a causa de la escala de análisis, que por abarcar una superficie de varios millones de kilómetros cuadrados de espacios fronterizos, se hace muy difícil el acceso a la información para un efectivo tratamiento de casos concretos. El estudio en profundidad de procesos transfronterizos como los que aquí se presentan e incluso la validación del enfoque propuesto para el análisis de las interacciones quedará para futuras investigaciones.

\section{CONSIDERACIONES PRELIMINARES. ESPACIO GEOGRÁFICO Y FRONTERAS}

El espacio entendido como construcción social es a la vez condición y soporte, por las construcciones y los recursos naturales que hacen posible la realización de las actividades y es también ámbito de disputa entre grupos y clases sociales.

Los elementos del espacio pertenecientes a los sistemas naturales constituyen medios de trabajo (tierra) y objetos de trabajo (agua, suelos, vegetación, fauna, minerales y fuentes de energía), por tanto oferta material del medio natural para la realización de actividades y para la reproducción del sistema en su totalidad. A estas se añade la oferta del medio construido, en forma de capital fijo (vías de circulación, almacenes, puertos, equipamientos e infraestructuras, servicios) como medios de trabajo y como valores de uso colectivo.

El espacio geográfico es por lo tanto, factor de producción, es recurso, junto al trabajo, el capital y la tecnología (Méndez, R. 2008); es medio de producción en tanto espacio de extracción de recursos y de realización de procesos de trabajo; por lo tanto se lo considera como fuerza productiva estratégica (Sánchez, J.E. 1991, Leff, E. 2003).

Esta primera consideración permite orientar los postulados de los apartados siguientes, en torno a los procesos de construcción de espacios fronterizos en particular en el MERCOSUR y el papel de estos procesos en tanto productores de desarrollos geográficos desiguales y, en segundo lugar, centrar el conjunto en el escenario actual de la crisis.

El territorio, como parte del espacio geográfico concreta, traduce un modo de recorte y de control del espacio que garantiza la especificidad y la permanencia, la reproducción de los grupos humanos que lo ocupan, es su dimensión política, que ilustra la naturaleza intencional 
y el carácter voluntario de su creación. El territorio implica siempre una apropiación del espacio.

Como noción jurídica, reenvía a la existencia del Estado, cuya legitimidad se mide en gran parte por su capacidad de garantizar la integridad territorial. El Estado asegura no solamente una autoridad territorial sobre un espacio delimitado y reconocido, al interior por toda la población y al exterior por los otros Estados y se encarga del ordenamiento del territorio y la regulación de las actividades delegando una parte de esta actividad a las instancias regionales.

Estas precisiones en torno al espacio geográfico y sus recursos naturales como fuerza productiva, así como los procesos de expansión geográfica como los más fuertes en términos de equipamiento de territorios para la realización de actividades pueden contribuir al mejor entendimiento de procesos sobre los espacios fronterizos a lo largo de la historia, en la actualidad y permitiría a la vez identificar tendencias y condicionantes para la puesta en marcha de alternativas a futuro.

\subsection{ENFOQUES EN TORNO A LAS INTERACCIONES FRONTERIZAS}

Aparte de la llamada "horogénesis" como enfoque para el estudio de la creación de fronteras en el mundo (Foucher, M, 1991) existen varias maneras de explicar de qué modo fueron y son construidos los espacios fronterizos.

Una manera surge desde la historia y los procesos de delimitación, demarcación, ocupación/apropiación y organización de los espacios de las fronteras, analizando de manera comparativa las dinámicas diferenciadas o no a uno y otro lado, tomando como eje central las asimetrías en los procesos, resultantes de la aplicación de políticas nacionales diferenciadas. Este enfoque se encuentra entre los primeros que fueron desarrollados en el campo de estudios fronterizos, como la larga serie de estudios de fronteras entre México y Estados Unidos o en el caso europeo, entre otros. En el Cono Sur se refleja en análisis particularmente centrados sobre las fronteras argentinas-uruguayas elaborados desde el Banco Interamericano de Desarrollo y su Instituto para la Integración de América Latina (BID-INTAL) a inicios de los años 90 (entre otros, González Posse, E. 1990, González Posse, E. Bolognesi Drosdoff, M.C; Iturriza,J y Valenciano E. 1990).

Otra manera puede ser partir del estudio de procesos transfronterizos, que surgen o llegan, atraviesan o no las fronteras, analizando los flujos de factores de producción (capital en forma de bienes, mercancías y servicios y financiero, trabajo y personas) en diversas escalas (Paelink, J, 1968; Alegría Olazábal, T, 1992; Otero-Schafer, N 1993). En este sentido se estudian las dinámicas en marcha de uno y otro lado y las maneras en que las mismas permean o no las fronteras, donde sobresalen enfoques teórico-metodológicos de Raffestin, solo para citar algunos (Raffestin, C. 1993). Derivados de estas aproximaciones se encuentran trabajos como los realizados sobre la Triple Frontera entre 1995 y 2004 por el autor, 
condensados en una publicación posterior en la que se analizaron las magnitudes, frecuencias y escalas así como el carácter transnacional/transfronterizo o estrictamente nacional en los flujos de bienes, personas y capitales (Schweitzer, A, 2009).

Una tercera posibilidad podría ser estudiar las maneras en que se diferencian los procesos de generación de valor y su apropiación en forma de excedente por parte de los actores participantes en procesos productivos que suceden, llegan o atraviesan las fronteras. En este sentido podría intentar explicarse a partir del estudio de subsistemas de acumulación (Rofman, A, 1984 y 1999; De Jong, G. 2009) y analizar los procesos de generación de desarrollos geográficos desiguales en espacios transfronterizos. Se trata de un enfoque que si bien se vincula con los precedentes y en particular con el anteriormente nombrado (análisis de flujos de factores de producción) se encuentra todavía sin demasiados antecedentes en estudios de frontera.

En manera muy sintética estos autores definen a los subsistemas (o sistemas) de acumulación como los encadenamientos necesarios para la realización de una actividad productiva concreta, ya sea a nivel de rama de la producción o de una cadena de valor específica, y apuntan a estudiar, en estos encadenamientos, las maneras en que cada paso en los mismos es realizado por los mismos o diferentes actores y por consiguiente, se apropian de manera diferencial de los excedentes generados a lo largo del proceso de producción, distribuyéndolos al interior del mismo proceso o invirtiéndolos en otros complementarios con fines de ampliar la reproducción del capital o transfiriéndolos hacia otros espacios como remesas de capital.

La integración analítica de este enfoque con el análisis de flujo de factores de producción podría aportar a darle mayor sentido a la interpretación de las maneras en que los excedentes obtenidos en espacios externos a las regiones fronterizas se distribuyen en segmentos de cadenas de subsistemas de acumulación que se realizan y atraviesan fronteras, y entender qué parte de estos excedentes se transforma en capital fijo mediante inversiones públicas y del sector privado. Asimismo permitiría analizar las transferencias de excedentes de los trabajadores al capital, del Estado al capital y al exterior, en escalas locales transfronterizas.

Estos enfoques podrían ayudar a profundizar estudios de casos históricos como por ejemplo los subsistemas transfronterizos ganaderos en Patagonia austral, con sede inicialmente en Malvinas, luego en Punta Arenas y finalmente en Buenos Aires o del Uruguay y las pampas gaúchas, en torno a Sant 'Ana do Livramento, en sus comienzos direccionados desde Montevideo antes de la construcción del puerto y ferrocarril hacia Río Grande, ambos entre 1880 y mediados de la década de 1910. Asimismo permitiría profundizar análisis de expansión de la soja en Paraguay en el marco del frente pionero gaúcho y la posterior expansión paranaense, con producción de plusvalor en Paraguay, por migrantes al menos originalmente brasileros, primero colonos y luego empresarios, apoyados por créditos obtenidos en bancos en el lado brasilero. 
Este enfoque también pueden ayudar a explicar la actualidad de la explotación de recursos naturales estratégicos (minería, petróleo, agua virtual) en espacios fronterizos del Cono Sur, en el escenario actual de ya más de dos décadas de políticas activas de integración regional, tema que es objeto de los apartados siguientes.

\subsection{FRONTERAS Y EXPANSIONES GEOGRÁFICAS}

La noción de expansión geográfica permite estudiar las maneras en que las dinámicas mundiales se despliegan hacia espacios que son nuevos o hacia espacios donde las actividades productivas y las sociedades se asientan en sectores menos dinámicos. Se trata de la adecuación de un territorio a los fines de asegurar las condiciones para la realización de nuevas actividades o de reconversión de las mismas bajo nuevas tecnologías o formas organizativas.

Estos procesos incluyen tanto la apropiación y control de territorios como la implantación de equipamientos e infraestructuras y la atracción de fuerza de trabajo como empleo directo o indirecto, para asegurar la reproducción de lo necesario y la realización del proceso de producción, de consumo productivo y la reproducción de la fuerza de trabajo. Esto obedecería a una fase de exportación de capitales, en forma de excedentes hacia nuevos espacios a fin de equiparlos y hacerlos funcionales con requerimientos definidos en los centros mundiales, de manera de mantener altas tasas de ganancia (Harvey, D, 2004).

Si consideramos este camino de expansión geográfica de manera combinada con la situación actual del desarrollo del espacio geográfico y los problemas ambientales descriptos en apartados anteriores, se facilita la comprensión de conceptos clave para entender porqué las actuales dinámicas mundiales de disputa por los recursos naturales tienen raíces históricas y ocasionan conflictos no solamente ambientales sino también y fundamentalmente, sociales.

Esto nos permite asimismo entender de qué manera, en la medida que se fueron implantando las actividades productivas a cada lado de las fronteras nacionales, en espacios fronterizos con mayor o menor grado de integración, se fueron produciendo diferentes formas de ocupación, organización y administración de los territorios.

La implantación de actividades en espacios nacionales o binacionales en escalas regionales, locales y transfronterizas se dará en la medida que las condiciones de producción necesarias para la realización de un determinado sistema de acumulación, como la yerba mate, la soja o los cultivos con fines alimentarios, solo para mencionar algunos, se encuentren a uno u otro lado de la frontera.

Estos procesos de formación de espacios transfronterizos de acumulación de capital dependerán a su vez de otras dos condiciones. La primera de ellas será de las coyunturas de las relaciones binacionales en la región le impusieran a las fronteras roles de barrera, filtro o libre circulación de factores. La segunda, de la forma en que los procesos de expansión geográfica, que por depender en gran parte de políticas públicas exigen la presencia de los 
estados nacionales o regionales, sirvieran de apoyo o no a la realización de esos procesos de acumulación.

Lo anterior, al menos a manera de hipótesis, podría explicar por qué en las fronteras de la Patagonia, con espacios muchas veces complementarios, o en los espacios de la Cuenca del Plata, donde situaciones complementarias se alternan con otras en las que de uno y otro lado de la frontera se dan similares condiciones para realización de actividades, los resultados en términos de desarrollo terminan siendo de tales niveles de desigualdad.

De este modo, las llamadas "asimetrías" fronterizas no se deberían solamente a oscilaciones cambiarias, a condiciones como las dos mencionadas arriba o a dinámicas demográficas y económicas diferenciales sino también a las distintas intensidades en que se efectivizaron sobre los territorios los procesos de expansión geográfica que permitieron la realización de esas actividades, esas procesos de atracción de población y capital fijo necesarios para la puesta en funcionamiento de los sistemas de acumulación de capital.

Los diferenciales en condiciones de vida de las poblaciones en estos espacios dependerán por consiguiente no solo de las políticas públicas nacionales sino también de las maneras en que los distintos actores intervinientes en cada uno de los subsistemas de acumulación de capital participan en el reparto del excedente generado, a lo largo de los encadenamientos productivos.

Esto puede estudiarse no solo para circuitos transfronterizos tradicionales como los de la producción de alimentos y encadenamientos de sistemas como los electrónicos, comestibles elaborados y productos textiles intercambiados en las redes de comercio de frontera formal o informal en forma de contrabando. También pueden analizarse para los casos de recursos naturales estratégicos como los ya mencionados de la minería metalífera, el petróleo y la exportación de agua virtual, donde los actores principales son Estados nacionales y empresas trasnacionales. Si en los primeros de estos sistemas de acumulación, basta para su realización la construcción de puentes y facilitación del cruce fronterizo, muchas veces en escalas locales, vecinales, en los segundos, las condiciones dependen de la puesta en marcha de dispositivos de mucha mayor envergadura, como el caso de los puertos marítimos y los corredores de transporte y energía de escala continental que actualmente alcanzan su mayor explicitación el la Iniciativa de integración de las Infraestructuras de Redes Sudamericanas (IIRSA) (Schweitzer, A y Schweitzer, M, 2009).

\section{FRONTERAS, CRISIS Y RECURSOS NATURALES EN EL SIGLO XXI}

La etapa mundial actual es consecuencia del pasaje a un periodo de especulación financiera iniciado en los años 70 del siglo pasado y que alcanza sus momentos más álgidos en la crisis desatada a partir de 2008. El escenario actual de constante expansión económica de potencias demográficas como China o India pone en el centro del debate temas tales como 
si estamos frente a un cambio del liderazgo mundial sin salir del sistema o si estamos experimentando los primeros pasos de una crisis sistémica, final o no, del propio capitalismo.

Son diversas las interpretaciones sobre el carácter y alcance de la crisis. En términos generales a partir de las modalidades de intervención de los Estados en el salvataje de bancos y en mucho menor intensidad en la preservación del empleo, existe el consenso en torno al final de la vigencia del capitalismo de signo neoliberal y de la unipolaridad y el pasaje posible a un mundo multipolar en el que potencias emergentes como China, la India o Rusia e incluso Brasil ocupen puestos de primer orden.

Potencias como China continúan teniendo los índices de crecimiento de los más altos del planeta. En este país, al igual que la India aunque en menor grado, las políticas públicas que apuntan al desarrollo del mercado interno están generando fuertes incrementos en las demandas de recursos naturales minerales, energéticos y alimentarios. Mientras esta demanda se mantenga en ascenso, el escenario mundial se orientará en dirección a estos inmensos captadores de flujos de recursos financieros y naturales, en sus roles de motores del capitalismo.

Otra crisis que está en avance acelerado es la ecológica, o más precisamente, geográfica que se expresa por las consecuencias del sobreconsumo de bienes materiales que amplía la huella ecológica y los efectos de calentamiento global, en la profundización de la acentuación de las desigualdades en el acceso a recursos básicos por amplios sectores de la sociedad y la creciente sucesión de catástrofes naturales. No son ajenas a esta crisis las consecuencias de la expansión geográfica de las grandes transnacionales hacia las periferias y el incremento en la extracción de recursos naturales.

Las dos, la de las altas finanzas y la ecológica-geográfica, serían dos lados de una sola crisis. En ambos casos se avanza sobre las condiciones de vida de la población mundial, por deterioro y pérdida de recursos alimentarios por competencia con otros usos como ser los biocombustibles, por desposesión de valores de uso colectivo como en el caso de los equipamientos e infraestructuras por parte de las empresas y en términos más globales, al deterioro de las condiciones ambientales mínimas como el acceso al suelo, alimentos, aire puro y calidad de agua.

\subsection{EL LUGAR DE LOS RECURSOS NATURALES COMO FACTORES ESTRATÉGICOS}

El periodo que se abre con la crisis estaría alterando significativamente el escenario mundial. Todo indicaría que en caso de no producirse graves alteraciones en la marcha del sistema capitalista, estaríamos en las puertas de una nueva fase de expansión geográfica en el marco de la acumulación de capital por las potencias emergentes, en el marco de una competencia por el liderazgo mundial en la nueva etapa y, en este caso se intensificarían aun 
más los efectos de la crisis ecológica-geográfica y los retrocesos en las condiciones de vida de la población, profundizando la huella ecológica global y generando nuevas desigualdades ${ }^{3}$.

En cuanto al futuro liderazgo, lo que importa es saber no sobre quién o quienes recaerá sino reflexionar y tomar consciencia del papel que le tocará a América Latina y el Cono Sur en particular si no media un cambio en los actuales modelos de desarrollo dominantes. Samir Amin planteaba en 2003 que en este inicio de siglo la hegemonía mundial se dirime en torno al dominio o control de cinco monopolios (Amin, S, 2003):

- monopolio de los flujos financieros, que permite incidir sobre las corrientes de capital e incidir en parte de la apropiación de excedentes de pueblos y estados periféricos hacia empresas y estados centrales;

- control de los medios de comunicación masiva en tanto difusores de pautas culturales y acceso a la información;

- monopolio o primacía en la investigación y desarrollo, a partir de los cuales se alcanzaría por ejemplo a nuevas y mejores herramientas para extracción de recursos o desarrollo de nuevos procesos productivos;

- $\quad$ monopolio de las armas de destrucción masiva, al menos en su rol disuasivo; y

- control del acceso a los recursos naturales, fundamentalmente mineros, energéticos y agua.

De estos cinco monopolios, verdaderos factores de poder en la actualidad, el último, relacionado en el entendimiento del espacio geográfico como fuerza productiva estratégica, es el único que solo se puede desplazar mediante su extracción o apropiación de territorios.

Para ilustrar el escenario que se abre con la crisis desatada en 2008, el caso del mercado del oro es emblemático. Entre ese año y 2010 el destino orientado a la inversión habría pasado de un $6.9 \%$ al $60 \%$ en 2009 estabilizándose en esos porcentajes. Estos destinos de resguardo de valor estarían explicados por el aumento de demanda de bancos europeos, particularmente alemanes y suizos, chinos e hindúes y explican a su vez el alza del precio y la nueva fiebre del oro (World Gold Council, 2010).

En la actualidad, en África grandes empresas y mineras chinas disputan recursos petroleros y mineros con capitales occidentales; en Asia central, financian la construcción de gasoductos y oleoductos para abastecer su creciente demanda que comienza a competir con la orientada a la provisión del fluido a Europa. En América latina, donde existe mucha mayor

\footnotetext{
${ }^{3}$ Existen diversos enfoques en torno al de huella ecológica en general originados en enfoques ecosocialistas. Expresa el uso desigual del espacio de reproducción social por parte de diferentes entidades territoriales. La huella ecológica se puede medir, sumando las hectáreas necesarias para cultivo, ganadería, forestación, vivienda y espacios marítimos pesqueros, denominadas hectáreas globales, utilizadas por los distintos países y ciudades en función del consumo material de sus poblaciones. En la actualidad los países del Norte ocupan un espacio mucho mayor que el de sus propios territorios, alcanzando unas ocho hectáreas globales por habitante, generando una huella ecológica sobre las economías más desprotegidas, que se encuentran en el límite de su espacio vital, con menos de dos hectáreas por persona (Pengue, W. 2002). En la actualidad la huella ecológica mundial es de 1,25 planetas (Boutaud, A y Gondran N, 2009).
} 
presencia de capitales europeos y estadounidenses, la reorientación de las exportaciones hacia el Este asiático podría preanunciar la llegada y el despliegue de capitales provenientes de ese "nuevo" espacio mundial.

\subsection{ACUMULACIÓN POR DESPOSESIÓN Y EXPROPIACIÓN DE RECURSOS NATURALES EN EL CONO SUR}

Hasta mediados de la década de 1990 los países del Cono Sur figuraban entre los grandes exportadores de recursos naturales en rubros tradicionales, agropecuarios y con escaso valor agregado, el denominado subsector "vegetativo" (Rofman, A. y Romero, L. 1998). Tampoco ocupaban lugares relevantes en los productos brutos internos de Argentina y Brasil, donde los modelos económicos venían orientados desde la década de 1930 hacia la Industrialización por Sustitución de Importaciones.

Hacia los años 80 del siglo pasado, como consecuencia de la aplicación de modelos neoliberales, consolidados una década después, comenzó a operar una reconversión económica en el conjunto de la región, por medio de la cual en pocos años se pasó de una estructura económica asentada en torno al modelo de industrialización sustitutiva hacia una base industrial en espacios centrales del bloque, con una muy extensa periferia primarioexportadora basada en la explotación de recursos naturales.

A diferencia del modelo similar que estuvo vigente en las primeras dos décadas del Siglo XX, el Cono Sur neoliberal de más de doscientos millones de habitantes se mueve en un escenario mundial donde el Estado, mucho más débil que en periodos anteriores, en algunos casos apenas reúnen condiciones para controlar y apropiarse de un mínimo de la renta producto de la aceleración de la extracción y exportación de excedentes y recursos naturales. En el caso argentino esa renta no se orienta siquiera a promover la recuperación de factores productivos, la industrialización y mejora real de condiciones de vida de la población, sino a garantizar a medias la estabilidad macroeconómica, retomar el pago de la deuda externa que había sido suspendido con la crisis de 2001 y asegurar a los capitales transnacionales la continuidad de la extracción de recursos naturales.

Estos procesos de "reconversión" se operaron mediante mecanismos de "acumulación por desposesión" o "expoliación" por los cuales empresas transnacionales se apropiaron de recursos naturales y empresas estatales en el marco de las privatizaciones (Harvey, D, 2004) como manera de reproducir y ampliar el capital, liberar activos, transferir excedentes de capital ocioso, desde espacios centrales a las periferias del sistema y recuperarlos obteniendo cada vez mayores dividendos.

En la Argentina este proceso de acumulación por desposesión se desplegó y continúa desplegándose, en dos pasos sucesivos: 1) 1975 y 2001, de generación de un circuito de excedentes de capital en dirección Norte-Sur + deuda externa + programas de ajuste estructural + desvalorización de activos + privatizaciones y 2) 2001 hasta la actualidad, de 
transferencia de excedentes del Estado a las empresas trasnacionales, mediante el circuito de devaluación del peso + baja de costos de extracción y exportación + debilidad de controles + baja de precios de la tierra + subsidios a empresas trasnacionales en un contexto de alza de precios internacionales (Schweitzer, A y Godoy, P 2010). En los restantes países del bloque, con algunos desfasajes temporales y mayores intensidades en Paraguay y Uruguay y tal vez menores en Brasil, se habrían operado procesos similares. En todos los casos, los espacios periféricos, coincidentes con los márgenes fronterizos, se encuentran entre los más afectados.

La flexibilización de legislaciones restrictivas sobre compra de tierras por extranjeros en fajas de frontera, la facilitación de la circulación de personas y capitales por disminución de funciones de control sobre fronteras internas del bloque y en general la profundización de la integración bajo signos neoliberales o condicionados por herencias de períodos de vigencia de ese modelo, aun no superadas, se encuentran entre otros mecanismos facilitadores de la expansión de nuevas actividades productivas primarias, extractivas o agroindustriales.

\subsection{RECURSOS NATURALES EN DISPUTA: ORO, PETRÓLEO, AGUA....}

En el período 1880 - 1912, en las secciones patagónicas de la Cordillera de los Andes, que hace frontera entre Argentina y Chile y aun antes de la firma de los acuerdos de delimitación de 1881 y 1902, regía el sistema de "Cordillera Libre" mediante el cual ambos países establecieron la libre circulación de factores de producción (lana, ovejas, capitales financieros y medios de trabajo en general, personas) permitiendo la expansión del frente ovino que en dirección Sur - Norte se extendía desde el polo de Punta Arenas hacia los litorales primero atlánticos y luego pacíficos en un espacio económico binacional de más de medio millón de $\mathrm{km} 2$, poco poblado pero fuertemente integrado en el mercado lanero mundial liderado por Londres. En la misma región pero sobre el litoral atlántico las Islas Malvinas, ocupadas y mediando el desalojo de la población local de argentinos y su reemplazo por migrantes de origen escocés, en 1833, por parte del Imperio Británico, fueron hasta 1877 el puente por el cual se extendió el circuito ovino hacia la Patagonia.

En 1907 el descubrimiento de petróleo en Comodoro Rivadavia preanunciaría el nuevo gran ciclo, una nueva etapa en la historia de la región, que se asentaría desde los años 70 sobre la apropiación de renta producto de la extracción de ese recurso no renovable. Poco después, en los años 90 y particularmente en los últimos años, a los hidrocarburos se les sumará la extracción de minerales metalíferos, particularmente oro y plata. El modelo primario, extractivo y exportador, donde el Estado apunta a apropiarse de parte de la renta solo cambiaría para empeorar, producto de la privatización de las mayores áreas petroleras y la cada vez menor presencia del Estado en la explotación. En el caso de los minerales es peor aún, la Ley de Minería de 1997 prohíbe al Estado participar en la actividad (ver figura 01).

En los espacios fronterizos de la cuenca del Plata, sus ríos inicialmente solo cumplieron el rol de vías de transporte predominantes, pero con la industrialización, desde las 
primeras décadas del Siglo XX, pasarán también a ser fuente de agua, de la misma manera que los espacios pampeanos proveedores de alimentos y los misioneros y chaqueños de provisión de bienes variados como maderas, yerba mate y otros productos.

A estos circuitos productivos se les sumarán ya en la década del 70 los aprovechamientos compartidos con fines de generación hidroeléctrica, donde destacan en orden cronológico Salto Grande, Itaipú y Yaciretá ${ }^{4}$.

Actualmente, a lo largo de la Cordillera de los Andes se encadenan yacimientos de minerales metalíferos, particularmente oro y plata, al punto que en 1997 los dos países establecieron un territorio minero binacional mediante el Tratado de Integración y Complementación Minera entre Argentina y Chile, con territorios delimitados y normas propias en términos impositivos, financieros, de libre circulación de capital físico y personas. Una nueva versión de la cordillera libre, en este caso específica para una actividad, se establece esta vez bajo las banderas canadiense, australiana, sudafricana y de los Estados Unidos de América.

A su vez, debido a la escasa concentración del mineral, las modalidades de explotación son a cielo abierto, con uso de cianuro, en emprendimientos de grandes magnitudes en términos de movimiento de tierras, infraestructura y equipamientos: la llamada megaminería.

Uno de los emprendimientos más significativos por sus implicancias territoriales es Bajo La Alumbrera, en el Noroeste de Argentina, que posee no solamente las instalaciones propias al yacimiento sino también un ferrocarril y terminal portuaria en la Hidrovía Paraguay-Paraná, abarcando tan solo en el despliegue espacial de la actividad a más de cinco provincias argentinas. Otro caso, situado en alta montaña es el de Pascua Lama, emprendimiento de la empresa Barrick Gold, de capitales canadienses, en territorio argentinochileno comprendido en el territorio del tratado binacional. Este emprendimiento de extracción de oro, plata y cobre, en caso de concretarse, pondría en riesgo glaciares y fuentes de agua en la región chilena de Atacama y la provincia argentina de San Juan, en ambos casos en espacios considerados entre las más secos de los dos países.

En los últimos años comienzan a percibirse las consecuencias negativas de la actividad en términos ambientales y de competencia por acceso a recursos agua, energía y suelos, en un marco de casi nula reinversión, muy baja generación de empleo calificado y muy escasos recursos aportados al sector público.

El subsistema de acumulación vinculado a la minería no ha sido aun estudiado, al menos en lo que respecta a la Argentina y Chile. Sin embargo, medidas como las adoptadas en Patagonia de promoción a las exportaciones por puertos patagónicos, que estuvo en vigencia entre 1997 y 2007, de exención impositiva para la importación de maquinarias necesarias para la explotación y el propio tratado minero binacional dejan entender que la

\footnotetext{
${ }^{4}$ También deben considerarse, por su influencia con lo que ocurre agua abajo en los mismos ríos, los aprovechamientos brasileños sobre los ríos Paraná y sus principales afluentes, particularmente el Tieté.
} 
mayor parte del valor producido, por lo tanto la mayor parte del excedente, se transfiere al exterior. En el caso de la propia cadena de valor, todos los insumos, maquinarias, herramientas y vehículos de transporte son provistos por empresas extranjeras que, de este modo, participan en la producción apropiándose de parte del excedente. El destino final de lo extraído es a su vez negociado en las casas matrices de las empresas, demostrando que en su totalidad se trata de un proceso no solo de exportación de recursos naturales sino también de trabajo calificado e importación de pasivos ambientales y contaminación.

Fig. 01. Bacías de hidrocarburos, mineracion, hidroelétricas, aquíferos e "campos de hielo" en Patagonia

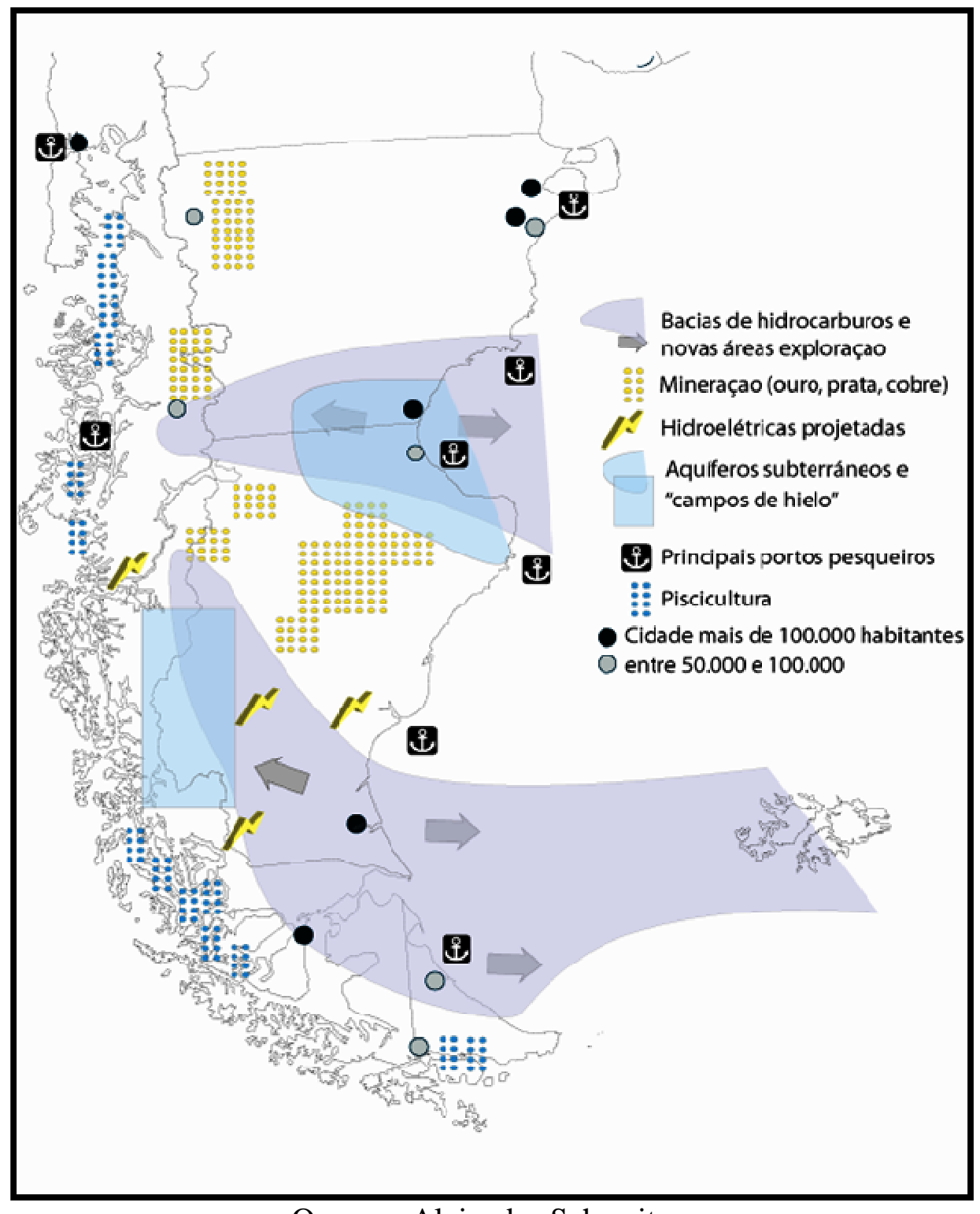

Org. por Alejandro Schweitzer

En Chile, donde el Estado participa en la explotación, el sector público se garantiza una apropiación al menos pequeña del excedente generado por empresas que en algunos casos 
son propiedad del mismo Estado mientras que, en Argentina, donde el Estado tiene prohibida su participación, el excedente apropiado por el mismo es ínfimo, de un 3\% como máximo del valor declarado a boca de mina.

Del lado de las condiciones laborales de los trabajadores y poblaciones locales, si bien los salarios pagados son relativamente altos en proporción a los percibidos por otros pobladores de las áreas cercanas a las explotaciones, el reciente accidente de la Mina San José en el Norte de Chile, así como los casos de contaminación de trabajadores por falta de medidas de prevención sucedidos en Huevos Verdes en Santa Cruz, en la Patagonia argentina, ocultados a la prensa y el derrame de ductos en Bajo la Alumbrera en el Noroeste del mismo país, que prueban ya la ausencia de voluntad de las empresas para paliar pasivos ambientales aun en etapas de explotación, evidencian una muy escasa participación de los mismos en la apropiación de valor, por lo tanto, una transferencia de excedentes extraordinaria de los trabajadores a las empresas.

Argentina y Brasil y en menor grado Chile cuentan con campos petroleros en diverso grado de explotación. En Argentina, la mayor proporción de la extracción se concreta en cuencas situadas en tierra firme. En los tres casos la extracción se realiza en modalidad off shore, sobre las respectivas plataformas marítimas, por lo tanto, en espacios fronterizos.

En Brasil, donde pese a situarse en fronteras, por limitar exclusivamente con aguas internacionales no existe presencia nacional alguna del otro lado, la situación fronteriza no presentaría problemas. En el mismo caso se encuentran las explotaciones en marcha en la cuenca de San Jorge en su porción off shore, compartida por las provincias de Chubut y Santa Cruz, en Patagonia austral, pero no sucede lo mismo en espacios marítimos de Argentina y Chile en el encuentro del Estrecho de Magallanes y el Mar Argentino, bajo jurisdicción chilena y argentina respectivamente.

En todos estos casos, aun en espacios en los que las fronteras marítimas se encuentran, como en el mencionado al final del párrafo anterior, los respectivos subsistemas de acumulación evidencian una diversa participación de capitales nacionales y transnacionales, dependiendo el país de que se trate. En los casos chileno y brasileño, donde las mayores empresas son de capital estatal, gran parte del excedente es apropiado por el propio Estado, mientras que en Argentina, pese a las condiciones de evidente saqueo del recurso, la exportación de hidrocarburos recibe una retención cercana al $44 \%$ del valor extraído a boca de pozo. Los niveles de equipamiento de puertos especializados, de apoyo a la actividad, muestran diversos niveles de "derrame" hacia sus áreas de influencia cercanas.

En la actualidad el problema de mayor importancia respecto a la explotación de hidrocarburos en espacios marítimos se relaciona con el descubrimiento de petróleo en la plataforma de las Islas Malvinas, en aguas de jurisdicción argentina en espacios próximos a la Zona de Exclusión establecida unilateralmente por la potencia ocupante. Este hecho volvió a poner en evidencia la confluencia de espacios fronterizos y en disputa y los recursos naturales. Al menos dos empresas de origen británico habrían anunciado el próximo inicio de la 
explotación. Frente a esta situación la Argentina tomó en 2010 la decisión de prohibir toda venta de equipos petroleros que pudieran ser destinados a la extracción en aguas de Malvinas, por lo que el inicio de la explotación demandaría a las empresas a realizar fuertes inversiones y al gobierno inglés a impulsar un significativo proceso de expansión de capitales sobre las Islas. Este proceso comenzó por la instalación de mayores contingentes militares y el envío de un submarino nuclear, también a inicios de este año ${ }^{5}$.

En la actualidad espacio de cultivo predominante de soja en grandes superficies de Argentina, Brasil y Paraguay y mayor diversificación en las porciones misioneras de los tres países, el espacio fronterizo ocupa el epicentro del acuífero Guaraní, junto al occidente del Uruguay y el oriente boliviano.

El subsistema de la soja se caracteriza por el casi completo control de la cadena productiva por parte de empresas transnacionales. En Argentina, luego de las primeras décadas dedicadas exclusivamente a soja, con gran pérdida de nutrientes, se adoptan sistemas de rotación trigo/soja, mediante el sistema de siembra directa, con mayor presión ambiental y uso de agroquímicos (Naumann, M. y Madariaga, M. 2003).

A estos mecanismos de degradación, erosión y contaminación se les suma el reemplazo de una producción agropecuaria orientada a la alimentación por cultivos agroindustriales asentados en las oleaginosas en general y la soja en particular, orientados a la producción y exportación de biocombustibles, aceites y harinas. El sistema apunta en general a la captura de agua, en un mecanismo que no apunta a la exportación del recurso sino a su consumo in situ, agregando por lo tanto no solo la pérdida y degradación del recurso agua sino también del suelo y sus nutrientes, en una de las mayores operaciones de aprovechamiento y exportación de agua virtual del planeta (Pengue, W. 2008).

Vinculados directamente con la expansión del cultivo de la soja se da por un lado la ampliación de la frontera agropecuaria, el avance sobre espacios de bosques y monte natural, a uno y otro lado de las fronteras de la región. La deforestación y pérdida de nutrientes no solo actúan contra la sustentabilidad de la propia producción de soja sino que ocasionan graves problemas de alteración de velocidades de escurrimiento de las aguas, ocasionando desastres como fueron, por ejemplo, las inundaciones sufridas por la ciudad de Santa Fe, en 2003 y 2007. Asimismo a lo mencionado se suma el consecuente transporte de cada vez mayores cantidades de sedimentos hacia las principales vías de navegación y principalmente el transporte de residuos de productos químicos utilizados en los cultivos contaminando campos y fuentes de agua dulce aguas abajo, en todos los casos con implicancias transfronterizas tanto en las causas como en las consecuencias.

\footnotetext{
${ }^{5}$ Las bases de los Estados Unidos en Colombia, la reapertura del Comando sur de la armada de ese país y la base británica de las Islas Malvinas, representan la nueva estrategia de despliegue de la OTAN en el continente.
} 


\section{A MODO DE CONCLUSIÓN. LAS FRONTERAS COMO ESPACIOS DE ACUMULACIÓN Y DE RESISTENCIA}

Sin dudas y tal como se presentó a lo largo del trabajo, los grandes beneficiarios de la explotación de los recursos naturales son las empresas transnacionales. Para estos actores, la localización fronteriza de los recursos no les representa mayores problemas que los relativos, entre otros, al cruce de mercaderías, facilitado desde los inicios del proceso de integración regional en 1986 o la necesidad de acelerar el acceso a los espacios de explotación y de exportación, que, como ya se adelantó, estarían siendo considerados en el marco de la IIRSA o de obtener mayores beneficios impositivos, menores restricciones legales y de control, flexibilización de regulaciones ambientales.

Tal como se expuso en apartados anteriores y se afirma en el párrafo previo, los territorios comprendidos en el Tratado Minero Argentino-Chileno y los de la Cordillera de los Andes en general, así como las áreas de producción de oleaginosas y biocombustibles situadas sobre el acuífero guaraní, se constituyen en inmensos espacios de acumulación en escalas transfronterizas.

Se impone realizar, en estos espacios, estudios sobre las maneras en que actualmente se despliegan y actúan procesos transfronterizos, identificar no solamente los flujos de factores sino, fundamentalmente, los actores y sus formas de articulación en torno a los subsistemas de acumulación, de manera de aportar a reforzar acciones que permitan asegurar mayores niveles de apropiación de excedentes por los estados en sus diferentes escalas y las sociedades regionales y locales y una mejor distribución que beneficie las condiciones de vida de las poblaciones directamente afectadas por efectos negativos. Se impone también agilizar los mecanismos de articulación de acciones, armonización de marcos normativos y agilización de controles por parte de los Estados nacionales, lo que significa nuevos y cada vez mayores desafíos para el proceso de integración regional y para que el mismo, definitivamente, se oriente en beneficio de la integración de los pueblos.

En relación a las sociedades regionales, en términos generales podría afirmarse que son inexistentes los casos de articulación de movimientos de resistencia frente a los efectos negativos de la explotación de recursos naturales como los descriptos, se trate de escalas nacionales, regionales o locales. Al parecer son problemas que salvo contados casos, se restringen a espacios nacionales, como si aun persistiera la idea de que las afectaciones ambientales o los efectos sociales y económicos negativos no permearan a través de las fronteras.

No ocurre lo mismo en los casos de la resistencia a la megaminería transnacional o al monocultivo de soja y la exportación de agua virtual. En estos dos casos, desde 2006 existen instancias de articulación de movimientos sociales, fundamentalmente en la Patagonia, con sus inicios en el lado argentino en 2001. 
Ese año ejerció en ese país un rol de bisagra. La crisis de fines de ese año tuvo características diversas de crisis económica-financiera, social y política. Esta crisis hizo coincidir la consolidación de algunas experiencias de autorganización de movimientos sociales contra las privatizaciones de los años 90, la profundización del deterioro de condiciones de vida de grandes masas de población y las primeras consecuencias visibles de la segunda fase de acumulación por desposesión (privatizaciones, saqueo petrolero y minero, expansión de los agrocombustibles y la soja particularmente).

En el año 2002 surge en Esquel, Provincia de Chubut, la primera asamblea que ya desde su origen se reclama como ambiental, como expresión del descontento de la sociedad local frente a la implantación de emprendimientos mineros a cielo abierto en las cercanías de la localidad. En marzo de 2003 como fruto de una consulta popular con gran participación de la población y en la cual el NO a la mina fue aprobado por el $81 \%$ de los votantes y de la extensión del reclamo hacia el conjunto de la provincia, el Gobierno de Chubut dicta la primera ley prohibición de la minería a cielo abierto. Entre ese año y 2008 otras seis provincias dictaron leyes similares, en todos los casos consecuencia del surgimiento y accionar de los movimientos sociales ambientales que fortalecidos por el triunfo en Chubut comenzaban a consolidarse en gran parte del territorio nacional.

En la actualidad más de ciento cincuenta asambleas y grupos de autoconvocados a lo largo de todo el país y se articulan en la Unión de Asambleas Ciudadana, formada en 2006 (UAC, 2010). La UAC actualmente expresa lo más avanzado del movimiento ambientalista y contra el saqueo de los recursos naturales. Desde su inicio, la UAC articularía los reclamos ambientales y contra el saqueo tanto en espacios mineros como de los hidrocarburos y de los cultivos agroindustriales tanto por el uso de agroquímicos como por su destino a la exportación en contexto de penuria alimentaria, bajo consignas como "No al saqueo y a la contaminación. Si a la vida", "No a la Mina", "El agua vale más que el oro" o "No a los biocombustibles", entre otras. Hasta agosto de 2010 se realizaron trece encuentros de esta "asamblea de asambleas",

En el lado chileno casi paralelamente se fueron constituyendo las primeras asambleas y foros contra el saqueo y la contaminación, que podrían ser producto de una primera experiencia para enfrentar la construcción de dos represas sobre el río Baker y una planta de aluminio, que llevó por consigna "No a Alumysa".

En 2006 se realizaron los primeros foros transfronterizos en Patagonia Austral, integrando movimientos sociales con distinto grado de consolidación procedentes de ambos lados de la cordillera, proseguido en foros subsiguientes, con sede en Esquel. Asimismo

\footnotetext{
${ }^{6}$ La UAC, como asamblea de asambleas, no tiene una dirección unificada ni pretende tenerla, como tampoco son unívocas las posiciones públicas de la unión ni de los organismos integranres, pero participa por medio de delegaciones propias en actividades tales como las conferencias y debates sobre cambio climático, las actividades contra la Infraestructura de Integración de las Redes Sudamericanas o la Cumbre de los Pueblos y los Derechos de la Madre Tierra celebrada en Cochabamba en 2010, asumiendo progresivamente reivindicaciones cada vez más globales y por lo tanto políticas.
} 
pobladores de ambos lados participan en las manifestaciones y movilizaciones contra el saqueo y la contaminación independientemente del lado de la frontera en que se realicen.

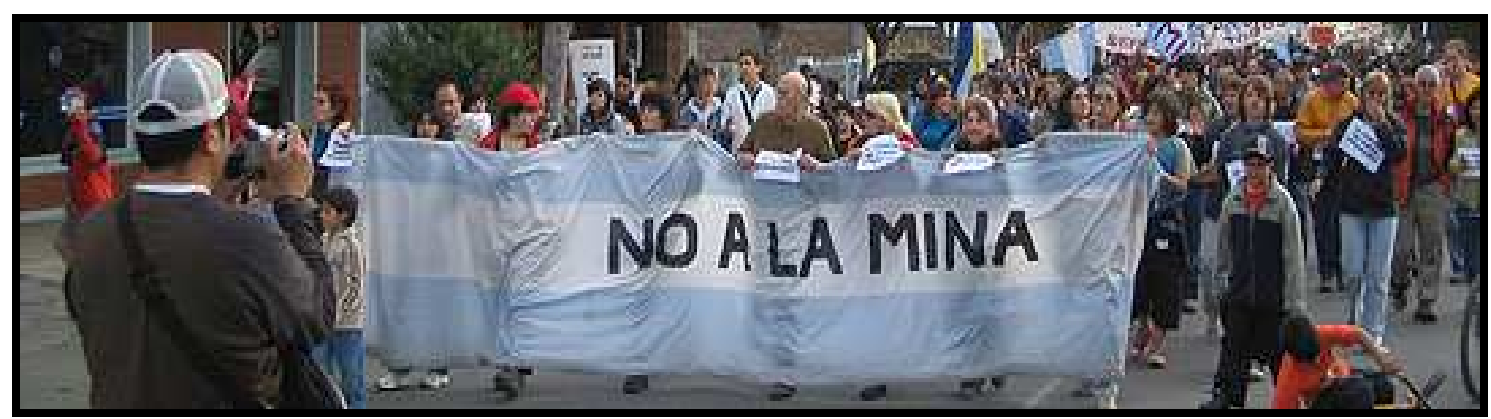

Movilización multisectorial, socialización de información y construcción de redes territoriales fueron los "factores clave" (Svampa, M, Solá Álvarez y Botaro, L. 2009) que favorecieron estos movimientos inicialmente dispersos.

Pasar del nivel actual de organización a su constitución en actores transfronterizos no es objetivo de movimientos como los mencionados y está lejos de hacerse realidad, al menos mientras solo en algunos casos encuentren contrapartes a los otros lados de las fronteras.

Sin embargo y de igual manera que lo señalado para las empresas, se impone en estos casos promover mayores niveles de participación, organización y articulación de las experiencias de los movimientos sociales en torno a programas comunes, a efectos de que se constituyan en sólidas instancias de integración de los pueblos.

\section{BIBLIOGRAFÍA}

ALEGRÍA OLAZÁBAL, Tito, 1992. Desarrollo urbano en la frontera México-Estados Unidos, Consejo Nacional para la cultura y las artes, México.

AMIN, Samir, 2003, Mas allá del capitalismo senil. Por un Siglo XXI no norteamericano, Paidós, Buenos Aires.

BOUTAUD, Aurélien y Gondran Natacha, 2009, L’empreinte écologique, La découverte, Paris.

DE JONG, Gerardo, 2009, Geografía, método regional y planificación, Catálogos, Buenos Aires.

FOUCHER, Michel, 1991, Fronts et frontières. Un tour du monde géopolitique, Fayard, Paris.

GONZÁLEZ POSSE, Ernesto; BOLOGNESI-DROSDOFF, María; Iturriza, Jorge y Valenciano, Ernesto, 1990, La frontera: un factor de integración, INTAL, Buenos Aires.

GONZÁLEZ POSSE, Ernesto, 1990, "Marco conceptual de la integración fronteriza promovida: las iniciativas de integración fronteriza", en Revista INTAL N¹56, BID-INTAL, Buenos Aires.

HARVEY, David, 2004, El nuevo imperialismo, Akal, Madrid.

LEFF, Enrique, 2003, Ecología y capital, Siglo XXI, México. 
MÉNDEZ, Ricardo, 2008, Geografía económica. La lógica espacial del capitalismo global, Ariel geografía, Madrid.

NAUMANN, Martín y Madariaga, Marta, 2003, Atlas argentino. Argentinienatlas, Secretaría de Ambiente y Desarrollo Sustentable - INTA - GTZ, Programa de Acción Nacional de Lucha contra la Desertificación, Buenos Aires.

OTERO-SCHAFER, Neiva, 1993, Urbanização na fronteIra. Expansão de Sant'Ana do Livramento, RS, UFRGS, Porto Alegre.

PAELINK, Jean, 1968, "Éléments pour l'étude fonctionnelle des régions-frontière", in Davin, L y Priebe, $\mathrm{H}$ (editores) Économie régionale sans frontières. Actes du Colloque Scientifique Belgo-Allemand d’économie régionale, Sciences et lettres de Liége, Liége.

PENGUE, Walter, 2002, "La Pampa sin el ombú...Comercio ecológicamente desigual y deuda ecológica" en Realidad Económica No 190. IADE. Buenos Aires.

PENGUE, Walter (compilador) 2008, La apropiación y el saqueo de la naturaleza. Conflictos ecológicos distributivos en la Argentina del bicentenario, Heinrich Böll - Gepama - Lugar, Buenos Aires.

RAFFESTIN, Claude, 1993, "Autour de la fonction sociale de la frontière", en Espaces et Sociétés Nº7071, Paris.

ROFMAN, Alejandro, 1984, "Subsistemas espaciales y circuitos de acumulación regional", Revista Interamericana de Planificación, Vol XVI, Nº2, Guatemala.

ROFMAN, Alejandro, 1999, Las economías regionales a fines del Siglo XX. Los circuitos del petróleo, del carbón y del azúcar, Ariel, Buenos Aires.

ROFMAN, Alejandro y Romero, Luís, 1998, Sistema socioeconómico y estructura regional en la Argentina, nueva edición actualizada. Amorrortu, Buenos Aires.

SÁNCHEZ, Joan Eugeni, 1991, Espacio, economía y sociedad, Siglo XXI, Madrid.

SCHWEITZER, Alejandro (2000) Aménagement du Territoire et Intégration Régionale dans le MERCOSUR: Frontières, Réseaux et Dynamiques Transfrontalières, ANRT, París/Lille.

SCHWEITZER, Alejandro (2009) "Dinámicas espaciales y territorios de la integración en el espacio transfronterizo del Iguazú", en Guibert, Martine et all (editores), Le Bassin du Rio de la Plata: intégration régionale et développement local, Presses Universitaires du Mirail, Toulouse.

SCHWEITZER Alejandro y Schweitzer Mariana, 2009, "La Integración de la Infraestructura Regional Sudamericana en la estrategia de extracción de recursos naturales del continente: El Eje Capricornio de IIRSA", presentado en el XII Congreso Internacional sobre Integración Regional, Fronteras y Globalización en el continente americano. Universidad de los Andes, San Cristóbal de Táchira, Venezuela. Mimeo.

SCHWEITZER, A y Godoy, P 2010 "Recursos naturales, ambiente y desarrollo sustentable en la patagônia austral. Los derechos humanos vistos desde las necesidades", presentado en las VII Jornadas de Historia Regional, en la Argentina del bicentenario, UNPA, Río Gallegos, mimeo. 
SVAMPA, Maristella, Sola Álvarez, Marian y Bottaro Lorena, "Los movimientos contra la minería metalífera a cielo abierto: escenarios y conflictos. Entre el "efecto Esquel" y el "efecto La Alumbrera", en Svampa, Maristella y Antonelli Mirta (editoras), 2009, Minería transnacional, narrativas del desarrollo y resistencias sociales, Biblos, Buenos Aires.

Unión de Asambleas Ciudadanas, http://asambleasciudadanas.org.ar/

World Gold Council, http://www.gold.org/ 